\title{
Organizational psychology on the way to 2065: a challenge to scholars
}

\author{
Richard E. Boyatzis * \\ Organizational Behavior, Case Western Reserve University, Cleveland, OH, USA
}

Keywords: organizational psychology, employee, leadership, interpersonal relationships, scholars

In the 48 years since I started in the field of Organizational Psychology (OP), a lot of interests have shifted or changed. Some are persistent, like leadership. Our fascination and fear with the forces behind change, innovation, and power continue to provoke research. We are less concerned about efficiency and less likely to study ways to drive people harder. Placement is less of a focus of research, in terms of selection and promotion. Employee participation has morphed from T-groups and sensitivity training, quality circles, employee involvement, business process re-engineering, and bottom up processes to engagement and citizenship. We have experienced philosophical shifts like the emergence of positive and spiritual psychology as disruptive innovations in thought. Some will be the phrenology of the future and pass away as ephemeral intellectual fads, and some will be extinction level events that dramatically change our perspectives and concepts of causality.

$\mathrm{OP}$ is becoming a leading integrative arena-truly transdisciplinary. This holds great promise for how our field can contribute into many other fields while maintaining rigor and developing our own concepts. Cleeremans (2010) challenged us to consider psychology a "hub" discipline of the fractionated and silo array of psychologies. In this same way, $O P$ can become an integrative hub for the social sciences. Our core intent is and will be to help create or maintain a better life for all in our societies, to seek truth, justice, beauty, and love (or at least get tenure).

There does seem to be eight trends emerging which, I believe, will affect the coming 30-50 years.

Edited and reviewed by: Axel Cleeremans,

Université Libre de Bruxelles, Belgium

${ }^{*}$ Correspondence:

Richard E. Boyatzis,

richard.boyatzis@case.edu

Specialty section:

This article was submitted to

Organizational Psychology,

a section of the journal

Frontiers in Psychology

Received: 23 March 2015

Accepted: 22 June 2015

Published: 07 July 2015

Citation:

Boyatzis RE (2015) Organizational psychology on the way to 2065: a challenge to scholars.

Front. Psychol. 6:948.

doi: 10.3389/fpsyg.2015.00948
I am not under the illusion that this list is comprehensive. Therefore, I ask the reader to use this list as their own call to intellectual curiosity to "go where no one has gone before" and help us create a desired future of rigorous and helpful insights through research in OP.

One set of trends address the "what" of a possible future OP:

(1) leadership beyond transformational styles (Bass, 1990) to characteristics like emotional and social intelligence in the context of $g$ and personality;

(2) development of people, dyads, teams, organizations, and communities beyond training to more contextual approaches that are integrated with work and life, like coaching (Van Oosten, 2013) which will integrate many forms of diversity of people and the importance of research on retained learning and what promotes it;

(3) interpersonal relationships (how we interact and effect each other, inclusiveness and diversity) beyond LMX (Graen and Uhl-Bien, 1995) like the concept of high quality connections (Dutton and Heaphy, 2003) shared vision (Clayton, 2014; Miller, 2014) and shared caring through compassion (Boyatzis et al., 2012); and

(4) using organizations to create a greater good and noble purpose in search of social justice, equality, intergroup relations, and well-being, like the work of Laszlo et al. (2014) in flourishing and sustainability, developing true measures of human resource and development profit and loss statements and balance sheets (not merely the financial rendering of them). 

OP:

The other set of trends address the "how" of a possible future

(5) holistic models and measures, using hormonal systems, neural networks, and nutrition as well as psychological and behavioral variables on effectiveness, mental health, and individual sustainability (Kahneman, 2011; Jack et al., 2012; Boyatzis et al., 2014; Waldman et al., 2014; Passarelli, 2015).

(6) complexity (i.e., non-linear, discontinuous, and emergent) beyond statistical manipulation to normalize the data but direct measures and analysis through mathematical modeling (Coen, 2013) and focusing on interpersonal dynamics (Hazy and Backström, 2013); and

(7) technological, social media, and disruptive innovations, like MOOCs (Massive On-Line Open Courses), social networks, and virtual reality as the basis for collecting research about these phenomena as well as the others mentioned above

\section{References}

Bass, B. M. (1990). From transactional to transformational leadership: learning to share the vision. Organ. Dyn. 18, 19-31.

Batista-Foguet, J. M., Revilla, M., Saris, W., Boyatzis, R. E., and Serlavós, R. (2014). Reassessing the effect of survey characteristics on common method bias in emotional and social intelligence competencies assessment. Struct. Equ. Model. 21, 596-607. doi: 10.1080/10705511.2014.934767

Boyatzis, R. E., Rochford, K., and Jack, A. (2014). Antagonistic neural networks underlying differentiated leadership roles. Front. Hum. Neurosci. 8:114. doi: 10.3389/fnhum.2014.00114

Boyatzis, R. E., Smith, M. L., and Beveridge, A. J. (2012). Coaching with compassion: inspiring health, well-being, and development in organizations. J. Appl. Behav. Sci. 49, 153-178. doi: 10.1177/0021886312462236

Clayton, B. (2014). Shared vision in corporate acquisitions. Front. Psychol. 5:1335. doi: 10.3389/fpsyg.2014.01335

Cleeremans, A. (2010). The grand challenge for psychology: integrate and fire. Front. Psychol. 1:12. doi: 10.3389/fpsyg.2010.00012

Coen, C. (2013). Relative performance and implicit incentives in the intergroup Prisoner's Dilemma. Organ. Behav. Hum. Decis. Process. 120, 181-190. doi: 10.1016/j.obhdp.2012.12.003

Dutton, J. E., and Heaphy, E. D. (2003). "The power of high quality connections at work," in Positive Organizational Scholarship:Foundations of a New Discipline, eds K. Cameron, J. Dutton, and R. Quinn (San Francisco, CA: Berrett-Koehler), 263-278.

Graen, G. B., and Uhl-Bien, M. (1995). Relationship-based approach to leadership: development of leader-member exchange (LMX) theory of leadership over 25 years: applying a multi-level multi-domain perspective. Leadersh. Q. 6, 219-247.

Hazy, J. K., and Backström, T. (2013). Human interaction dynamics (HID): foundations, definitions, and directions. Emergence 15, 91-111. and experimenting with new pedagogies and assessment of learning and development. This trend could have a useful impact on new pedagogy that might enhance learning (see trend $\# 2$ above).

(8) more appropriate and sensitive measurement of variables beyond single source studies to truly multi-method, multitrait designs using data from many sources like physiological and behavioral assessment. For example, discovery that a 5 or 7 point response set in surveys does not work reliably for European samples for whom all social measurement is on a 10 point scale (Batista-Foguet et al., 2014) is the kind of discovery that can change our assessments to be more contextually sensitive.

It is an exciting time of discovery and holds much promise for helping scholars find ways to share their findings and thoughts and create new knowledge.

Jack, A. I., Dawson, A., Begany, K., Leckie, R. L., Barry, K., Ciccia, A., et al. (2012). fMRI reveals reciprocal inhibition between social and physical cognitive domains. Neuroimage 66C, 385-401. doi: 10.1016/j.neuroimage.2012.10.061

Kahneman, D. (2011). Thinking, Fast and Slow. New York, NY: Farrar, Straus and Giroux.

Laszlo, C., Saillant, R., Cooperrider, D. L., and Brown, J. S. (2014). Flourishing Enterprise: The New Spirit of Business. Rosewood, CA: Stanford University Press.

Miller, S. P. (2014). Next-generation leadership development in family businesses: the critical roles of shared vision and family climate. Front. Psychol. 5:1335. doi: 10.3389/fpsyg.2014.01335

Passarelli, A. (2015). The neuro-emotional basis of developing leaders through personal vision. Fron. Psychol. 5:1335. doi: 10.3389/fpsyg.2014.01335

Van Oosten, E. (2013). The Impact of Emotional Intelligence and Executive e Coaching Onleader Effectiveness. Unpublished Ph.D. dissertation, Case Western Reserve University, Cleveland, $\mathrm{OH}$.

Waldman, D., Reina, C., and Peterson, S. (2014). "A neuroscience perspective of emotions in the formation of shared vision," in Paper Presented at the Annual Academy of Management Meeting (Philadelphia, PA).

Conflict of Interest Statement: The author declares that the research was conducted in the absence of any commercial or financial relationships that could be construed as a potential conflict of interest.

Copyright (C) 2015 Boyatzis. This is an open-access article distributed under the terms of the Creative Commons Attribution License (CC BY). The use, distribution or reproduction in other forums is permitted, provided the original author(s) or licensor are credited and that the original publication in this journal is cited, in accordance with accepted academic practice. No use, distribution or reproduction is permitted which does not comply with these terms. 